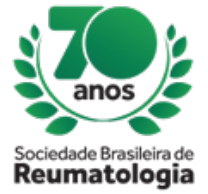

\title{
DISEASE ACTIVITY STATUS AND AND PHYSICAL FUNCTIONING IS ASSOCIATED WITH LOW MUSCLE MASS RELATIVE TO FAT MASS IN RHEUMATOID ARTHRITIS
}

Leonardo Peterson Santos (Serviço de reumatologia, hospital de Clínicas de Porto Alegre, universidade federal do Rio Grande do Sul, Brazil, Porto Alegre, RS, Brasil), Rafaela Cavalheiro Espírito Santo (Serviço de reumatologia, hospital de Clínicas de Porto Alegre, universidade federal do Rio Grande do Sul, Brazil, Porto Alegre, RS, Brasil), Jordana Miranda Souza Silva (Serviço de reumatologia, hospital de Clínicas de Porto Alegre, universidade federal do Rio Grande do Sul, Brazil, Porto Alegre, RS, Brasil), Joshua Baker (Hospital of the University of Pennsylvania, Estados Unidos), Vanessa Hax (Serviço de reumatologia, hospital de Clínicas de Porto Alegre, universidade federal do Rio Grande do Sul, Brazil, Porto Alegre, RS, Brasil), Lidiane Isabel Filippin (Serviço de reumatologia, hospital de Clínicas de Porto Alegre, universidade federal do Rio Grande do Sul, Brazil, Porto Alegre, RS, Brasil), Priscila Schmidt Lora (Serviço de reumatologia, hospital de Clínicas de Porto Alegre, universidade federal do Rio Grande do Sul, Brazil, Porto Alegre, RS, Brasil), Ricardo Machado Xavier (Serviço de reumatologia, hospital de Clínicas de Porto Alegre, universidade federal do Rio Grande do Sul, Brazil, Porto Alegre, RS, Brasil)

\section{BACKGROUND}

Rheumatoid Arthritis (RA) shows low appendicular lean mass index (ALMI) and higher fat mass index (FMI). The adiposity is an important confounder that may mask true relationships between physical functioning and ALMI. Our objective was to assess muscle mass relative to fat mass and verify associations this parameter with disease activity status, functional capacity and biological therapy.

\section{MATERIALS AND METHODS}

RA patients, aged between 40 and 70 years, were recruited and followed for 12 months. Body composition was assessed by total body dual-energy $\mathrm{x}$-ray absorptiometry for measurement of ALMI and FMI. ALMI values were adjusted for FMI (ALMI/FMI) and transformed in Z-Scores using a published method. Disease activity was assessed by Disease Activity Score-28 (DAS28) with protein C reactive and RA patients were divided in non-remission ( $>2.7$ ) and in remission (<2.7). Physical functioning was assessed by Health Assessment Questionnaire. Pharmacological treatment were assessed in patient medical records and the RA were divided in patients treated with biologic therapy (bDMARDs) and non-treated with bDMARDs. Frequency analysis, Pearson Correlations and GEE analyses were used and statistical significance was considered as $\mathrm{p}<0.05$.

\section{RESULTS}

Of 90 patients recruited, 81 completed the study (90\%). Most patients were women (88.9\%). The mean age was $56.5 \pm 7.3$ years and median disease duration was 8 years (IQR 3-18). At baseline, the median DAS28-CRP was 3.0 (IQR 1.0-3.0) and thirty percent of the RA patients were treated with bDMARDs. After 12 months, the DAS28-CRP and the use of bDMARD did not change ( $p>0.05)$. Eleven RA patients (12.2\%) showed low ALMI/FMI (Z-score $\leq-1)$ at baseline and 13 (16.0\%) after 12 months. After 12 months, ALMI/FMI was inversely associated with HAQ-DI ( $r=-0.3 ; p<0.05)$. The changes on ALMI/FMI was associated with disease activity status, where, patients in remission showed higher ALMI/FMI than patients not in remission Treatment with bDMARDs was not associated with changes in ALMI/FMI, however, the ALMI/FMI of RA patients treated with bDMARDs was numerically higher than patients not treated with bDMARDs.

\section{CONCLUSION}

Low skeletal muscle mass relative to adiposity was common in RA patients. This condition was associated with decreased physical function. Patients not in remission had lower muscle mass compared with those 
in remission; trend for further decrease in 12 months. Further studies are necessary to elucidate the direct impact of bDMARDs on body composition in RA patients. 